\title{
PRE-MODULAR CATEGORIES OF RANK 3
}

\author{
VICTOR OSTRIK
}

\begin{abstract}
We classify ribbon semisimple monoidal categories with three isomorphism classes of simple objects over the field of complex numbers.
\end{abstract}

\section{INTRODUCTION}

Let $k$ be an algebraically closed field of characteristic 0 . A fusion category $\mathcal{C}$ over $k$ is a $k$-linear semisimple rigid monoidal category with finitely many simple objects and finite dimensional spaces of morphisms, such that the endomorphism algebra of the neutral object is $k$, see [7. The rank of a fusion category is just the number of isomorphism classes of simple objects. In [15] we classified all fusion categories of rank 2. A similar classification problem for rank 3 seems to be out of reach at the moment. For example it is not known whether the number of fusion categories of rank 3 is finite. In this note we classify the fusion categories $\mathcal{C}$ of rank 3 under an additional assumption that $\mathcal{C}$ admits a ribbon structure, see [1. Recall that the ribbon fusion categories are called pre-modular, see [14.

Main Theorem. There are exactly 7 fusion categories of rank 3 admitting a structure of ribbon category.

The proof of this theorem is based on the properties of $S$-matrix (see 1]) and is an exercise in Galois theory. This note was inspired by [10 where the authors classified the fusion rings of modular tensor categories with small number of simple objects and small fusion coefficients via computer search. We expect that the list of modular categories of rank 4 contained in [10] is complete.Probably the methods of this paper are sufficient to prove this. It would be more interesting to prove some general finiteness theorems, for example to answer the following

Question. Is it true that there are only finitely many ribbon categories of a given finite rank?

This question is a special case of question in [15].

After this note was finished D. Nikshych showed to the author reference [2] where the results close to the results of this paper were obtained. Still we hope that our treatment will be useful for some readers.

This note was written while the author enjoyed the hospitality of the Institute for Advanced Study. I am happy to thank this institution.

\section{Recollections}

In this section we review necessary facts on pre-modular categories.

Date: March 2005.

The author was partially supported by NSF grant DMS-0098830 and DMS-0111298. 
2.1. Dimension. Recall (see e.g. [7) that a fusion category $\mathcal{C}$ is pivotal if it is endowed with a functorial tensor isomorphisms $M \rightarrow M^{* *}$ for any $M \in \mathcal{C}$. In a pivotal fusion category $\mathcal{C}$ one defines for any object $M \in \mathcal{C}$ its dimension $d_{M} \in k$, see [1. We have the following properties, see loc. cit:

(a) $d_{M}$ defines a homomorphism $d_{M}: K(\mathcal{C}) \rightarrow k$;

(b) Assume that $M$ is simple object. Then $d_{M} \neq 0$.

2.2. $\tilde{S}$-matrix. Let $\mathcal{C}$ be a ribbon category (see [1] for a definition). Recall that in a ribbon category the balancing isomorphism $\theta \in \operatorname{End}\left(\operatorname{Id}_{\mathcal{C}}\right)$ is defined. For a simple object $X \in \mathcal{C}$ let $\theta_{X}$ denote the scalar by which $\theta$ acts on $X$. Vafa's theorem (see [17, 1, 8]) states that

(a) the numbers $\theta_{X}$ are roots of unity.

Let $\left\{V_{i}\right\}_{i \in I}$ be a set of (reprsentatives of isomorphism classes of) simple objects in $\mathcal{C}$ and let $i \mapsto i^{*}$ be a unique involution of $I$ such that $\left(V_{i}\right)^{*} \cong V_{i^{*}}$. Let $V_{i} \otimes V_{j}=$ $\sum_{k \in I} N_{i j}^{k} V_{k}$ and let $\theta_{i}:=\theta_{V_{i}}, d_{i}:=d_{V_{i}}$. Define the matrix $\tilde{S}$ by the formula (see (1):

$$
\tilde{S}_{i j}=\theta_{i}^{-1} \theta_{j}^{-1} \sum_{k \in I} N_{i^{*} j}^{k} \theta_{k} d_{k} .
$$

We have the following properties, see 1]:

(b) the matrix $\tilde{S}$ is symmetric $\tilde{S}_{i j}=\tilde{S}_{j i}$;

(c) For any $i \in I$ the assignment $\phi_{i}\left(V_{j}\right)=\tilde{S}_{i j} / d_{i}$ defines a homomorphism of rings $K(\mathcal{C}) \rightarrow k$.

Recall (see [1]) that a ribbon category $\mathcal{C}$ is called modular if the matrix $\tilde{S}$ is non-degenerate.

\section{RIBBON BASED RINGS OF RANK 3}

3.1. Let $k, l, m, n$ be nonnegative integers subject to the condition

$$
k^{2}+l^{2}=l m+k n+1 .
$$

Let $K(k, l, m, n)$ be the based ring with the basis $1, X, Y$ and the multiplication given by

$$
X^{2}=1+m X+k Y, Y^{2}=1+l X+n Y, X Y=Y X=k X+l Y .
$$

The following Proposition gives the classification of the based rings of rank 3 , see 7], Example in section 8.10.

Proposition. Let $K$ be a based ring of rank 3. Then either $K=K(\operatorname{Rep}(\mathbb{Z} / 3 \mathbb{Z}))$ or $K=K(k, l, m, n)$.

Note that we have an obvious isomorphism of the based rings $K(k, l, m, n)=$ $K(l, k, n, m)$.

3.2. Symmetric categories. Recall that a ribbon category $\mathcal{C}$ is called symmetric if the square of the braiding is the identity. Equivalently, the $\tilde{S}$-matrix of the category $\mathcal{C}$ has rank 1 (see e.g. [14]). It is proved by Deligne [5] that for any symmetric fusion category $\mathcal{C}$ there exists a finite group $G$ and an equivalence $\mathcal{C} \simeq$ $\operatorname{Rep}(G)$.

Lemma. Let $G$ be a finite group with 3 irreducible representations. Then either $G=\mathbb{Z} / 3 \mathbb{Z}$ or $G=S_{3}$.

Proof. The Landau estimate (see [13, 15]) gives $|G| \leq 6$. The rest is easy. 
Corollary. Let $\mathcal{C}$ be a symmetric category of rank 3. Then either $K(\mathcal{C})=$ $K(\operatorname{Rep}(\mathbb{Z} / 3 \mathbb{Z}))$ or $K(\mathcal{C})=K(0,1,0,1)$.

3.3. Non-modular and non-symmetric categories. Assume that the category $\mathcal{C}$ is not symmetric and is not modular. It follows from [14] Corollary 2.16 that the category $\mathcal{C}$ has a non-trivial symmetric subcategory. This subcategory has 2 simple objects (say 1 and $X$ ) and thus is equivalent to $\operatorname{Rep}(\mathbb{Z} / 2 \mathbb{Z})$. Hence $K(\mathcal{C})=$ $K(0,1,0, n)$. Observe that $d_{X}=1$ since otherwise $d_{Y}=0$. Thus $\tilde{S}$-matrix looks like

$$
\tilde{S}=\left(\begin{array}{ccc}
1 & 1 & d_{Y} \\
1 & \theta_{X}^{-2} & \theta_{X}^{-1} d_{Y} \\
d_{Y} & \theta_{X}^{-1} d_{Y} & \theta_{Y}^{-2}\left(1+\theta_{X}+n \theta_{Y} d_{Y}\right)
\end{array}\right) .
$$

Since the second column should give a homomorphism $K(\mathcal{C}) \rightarrow \mathbb{C}$ we get $\theta_{X}=1$ (except, possibly, the case $n=0$ ). Since the third column is a homomorphism $K(\mathcal{C}) \rightarrow \mathbb{C}$ distinct from $d$ times $d_{Y}$ we get $\theta_{Y}^{-2}\left(1+\theta_{X}+n \theta_{Y} d_{Y}\right)=d_{Y} \bar{d}_{Y}$ where $\bar{d}_{Y}$ is a root of the equation $y^{2}=2+n y$ distinct from $d_{Y}$. Thus we have $\theta_{Y}^{-2}\left(1+\theta_{X}+\right.$ $\left.n \theta_{Y} d_{Y}\right)=-2$ or equivalently $n d_{Y}=-2\left(\theta_{Y}+\theta_{Y}^{-1}\right)$. Assume that $n>1$. Then $d_{Y}$ is irrational and after applying a Galois avtomorphism to the last equation we have $n y_{+}=-2\left(\theta+\theta^{-1}\right)$ where $y_{+}$is the positive root of the equation $y^{2}=2+n y$ and $\theta$ is a some root of unity. But note that $y_{+}^{2}>n y_{+}$and hence $y_{+}>n$ and $n y_{+}>n^{2}$. On the other hand clearly $\left|\theta+\theta^{-1}\right| \leq 2$. Thus $n^{2}<4$ and we get the contradiction. Thus we have proved

Proposition. Assume that $\mathcal{C}$ is nor symmetric neither modular. Then $K(\mathcal{C})=$ $K(0,1,0, n)$ where $n=0,1$.

Remark. It is reasonable to expect that if $K(\mathcal{C})=K(0,1,0, n)$ for some fusion category $\mathcal{C}$ then $n \leq 2$ (see Remark in Section 4.5). But unfortunately we don't know how to prove that $n$ is bounded by any constant.

3.4. Modular categories. In this section we assume that $\mathcal{C}$ is a modular category such that $K(\mathcal{C})=K(k, l, m, n)$. Let $\phi_{1}, \phi_{2}, \phi_{3}$ be the three distinct homomorphisms $K(\mathcal{C}) \rightarrow \mathbb{C}$; we assume that $\phi_{1}$ coincides with the dimension function and denote $\phi_{i}(X)=x_{i}, \phi_{i}(Y)=y_{i}$ for $i=1,2,3$. We can assume that $\tilde{S}$-matrix looks like

$$
\tilde{S}=\left(\begin{array}{ccc}
1 & x_{1} & y_{1} \\
x_{1} & x_{1} x_{2} & y_{1} x_{3} \\
y_{1} & x_{1} y_{2} & y_{1} y_{3}
\end{array}\right) .
$$

Since $\tilde{S}$-matrix is symmetric we have $x_{1} y_{2}=y_{1} x_{3}$. It is easy to see that $x_{1} y_{2}=$ $y_{1} x_{3} \neq 0$.

The absolute Galois group $\operatorname{Gal}(\overline{\mathbb{Q}} / \mathbb{Q})$ acts on the set $\left\{\phi_{1}, \phi_{2}, \phi_{3}\right\}$. Thus we have a homomorphism $\operatorname{Gal}(\overline{\mathbb{Q}} / \mathbb{Q}) \rightarrow S_{3}$. Let us denote the image of this homomorphism by $G$. It is known [4 that the group $G$ is abelian (see also [3, 7] Appendix). Thus we have 3 possibilities: $G$ is trivial, $G=\mathbb{Z} / 3 \mathbb{Z}$ and $G=\mathbb{Z} / 2 \mathbb{Z}$.

Case 1. $G$ is trivial. Then all numbers $x_{i}, y_{i}$ are rational and hence integer. In particular $\mathrm{FPdim}(X)$ and $\mathrm{FPdim}(Y)$ are integers. Then again the Landau estimate (see [7] 8.38) gives $\operatorname{FPdim}(\mathcal{C}) \leq 6$ and the only possibility is $K(\mathcal{C})=K(0,1,0,1)$.

Case 2. $G=\mathbb{Z} / 3 \mathbb{Z}$. The group $G$ permutes homomorphisms $\phi_{1}, \phi_{2}, \phi_{3}$ cyclically. Thus applying the elements of $G$ to the identity $x_{1} y_{2}=y_{1} x_{3}$ we get new identities $x_{2} y_{3}=y_{2} x_{1}$ and $x_{3} y_{1}=y_{3} x_{2}$. Equivalently $x_{1} y_{2}=x_{2} y_{3}=x_{3} y_{1}=: \lambda$. Recall that $\lambda \neq 0$. Thus $\left(x_{1}, x_{2}, x_{3}\right)=\lambda\left(y_{2}^{-1}, y_{3}^{-1}, y_{1}^{-1}\right)$. The numbers $x_{i}$ are the roots of the 
polynomial $x^{3}-(m+l) x^{2}+\left(m l-k^{2}-1\right) x+l$ (the characteristic polynomial of the operator of multiplication by $X$ in $K(k, l, m, n))$ and the numbers $y_{i}$ are the roots of the polynomial $y^{3}-(n+k) y^{2}+\left(n k-l^{2}-1\right) y+k$. The Vieta Theorem implies:

$$
\begin{gathered}
-l=x_{1} x_{2} x_{3}=\frac{\lambda^{3}}{y_{1} y_{2} y_{3}}=\lambda^{3} /(-k) \Rightarrow \lambda^{3}=l k \\
m+l=x_{1}+x_{2}+x_{3}=\lambda\left(y_{1}^{-1}+y_{2}^{-1}+y_{3}^{-1}\right)=\lambda \frac{n k-l^{2}-1}{(-k)} \\
m l-k^{2}-1=x_{1} x_{2}+x_{2} x_{3}+x_{3} x_{1}=\lambda^{2} \frac{y_{1}+y_{2}+y_{3}}{y_{1} y_{2} y_{3}}=\lambda^{2} \frac{n+k}{(-k)}
\end{gathered}
$$

Now equation (2) implies that $\lambda$ is rational except, possibly, the case $m+l=0$ (in the latter case $K(k, l, m, n)=K(1,0,0,0))$. Equation (1) then says that $\lambda>0$ and equations (2), (3) imply $n k-l^{2}-1<0$ and $m l-k^{2}-1<0$. But we know from $(*)$ that $n k-l^{2}-1+m l-k^{2}-1=-3$ and hence we can assume that $n k-l^{2}-1=-1$ and $m l-k^{2}-1=-2$. It is easy to see that these equations imply $k=1$ and $l=1,2$. But the case $l=2$ is impossible since then $\lambda=\sqrt[3]{k l}$ is irrational. Thus the only possibility is $K(\mathcal{C})=K(1,1,1,0)$. Thus we found that in case 2 we have 2 possibilities: either $K(\mathcal{C})=K(1,1,1,0)$ or $K(k, l, m, n)=K(1,0,0,0)$.

Case 3. $G=\mathbb{Z} / 2 \mathbb{Z}$. In this case there are two subcases:

(a) $G$ fixes $\phi_{1}$ and permutes $\phi_{2}$ and $\phi_{3}$. The identity $x_{1} y_{2}=y_{1} x_{3}$ implies $x_{1} y_{3}=y_{1} x_{2}$ and hence $x_{2} y_{2}=x_{3} y_{3}$. Thus $\phi_{2}(X \otimes Y)=\phi_{3}(X \otimes Y)$. Since $\phi_{2} \neq \phi_{3}$ we see that $(X Y)^{2}$ should lie in the subspace of $K(\mathcal{C})$ spanned by 1 and $X Y$. Now $(X Y)^{2}=(k X+l Y)^{2}=k^{2} X^{2}+2 k l X Y+l^{2} Y^{2}=k^{2}(m X+k Y)+l^{2}(l X+n Y)$ $\bmod <1, X Y>=\left(k^{2} m+l^{3}\right) X+\left(k^{3}+l^{2} n\right) Y \bmod <1, X Y>$. This vector should be proportional to $X Y=k X+l Y$, hence $\left(k^{2} m+l^{3}\right) l=\left(k^{3}+l^{2} n\right) k$. We see that if $p$ is a prime divisor of $k$ then $p$ divides $l$ and the relation $(*)$ then shows that $p$ divides 1 . Thus $k \leq 1$ and similarly $l \leq 1$. Thus in this case we have that either $K(\mathcal{C})=K(0,1,0, n)$ or $K(\mathcal{C})=K(1,1,1,0)$. In the first case we have $x_{1}=1$ (otherwise $y_{1}=0$ ) and $y_{1}$ is a root of equation $y^{2}=2+n y$. This equation has a rational root only for $n=1$. Thus we have 2 possibilities $K(\mathcal{C})=K(0,1,0,1)$ and $K(\mathcal{C})=K(1,1,1,0)$.

(b) $G$ does not fix $\phi_{1}$. This is most difficult case. We can assume that $G$ permutes $\phi_{1}$ and $\phi_{2}$ and fixes $\phi_{3}$. Thus the identity $x_{1} y_{2}=y_{1} x_{3}$ implies $x_{2} y_{1}=y_{2} x_{3}$ and hence $x_{1} x_{2}=x_{3}^{2}$. Thus by Vieta Theorem $x_{3}^{3}=x_{1} x_{2} x_{3}=-l$. Set $t:=x_{3}$ and $s:=y_{3}$; then $s$ and $t$ are integers. Assuming $s \neq 0$ we have

$$
k=s+t^{2} s, l=-t^{3}, m=t-t s^{2}-\frac{s^{2}+1}{t}, n=s+\frac{t^{4}-1}{s} .
$$

Also

$$
\begin{gathered}
x_{1} x_{2}=\frac{-l}{x_{3}}=t^{2}, x_{1}+x_{2}=m+l-x_{3}=-t s^{2}-t^{3}-\frac{s^{2}+1}{t}, \\
y_{1} y_{2}=\frac{-k}{y_{3}}=-\left(t^{2}+1\right), y_{1}+y_{2}=n+k-y_{3}=t^{2} s+s+\frac{t^{4}-1}{s} .
\end{gathered}
$$

The equation $x_{1} y_{2}=y_{1} x_{3}$ gives $x_{1}=y_{1}^{2} x_{3} /\left(y_{1} y_{2}\right)=-\frac{t}{t^{2}+1} y_{1}^{2}$ and, similarly, $x_{2}=$ $-\frac{t}{t^{2}+1} y_{2}^{2}$. Thus we have $x_{1}+x_{2}=-\frac{t}{t^{2}+1}\left(y_{1}^{2}+y_{2}^{2}\right)$ or, equivalently,

$$
-t s^{2}-t^{3}-\frac{s^{2}+1}{t}=-\frac{t}{t^{2}+1}\left(\left(s\left(t^{2}+1\right)+\frac{t^{4}-1}{s}\right)^{2}+2\left(t^{2}+1\right)\right) .
$$


After simple transformations we have

$$
\frac{s^{2}+1}{t^{2}}+t^{2}-s^{2} t^{2}-2 t^{4}=\frac{\left(t^{2}-1\right)^{2}}{s^{2}}\left(t^{2}+1\right)
$$

and thus

$$
\frac{s^{2}}{t^{2}}\left(1-t^{4}\right)+\frac{1}{t^{2}}+t^{2}-2 t^{4}=\frac{\left(1-t^{2}\right)^{2}}{s^{2}}\left(t^{2}+1\right) .
$$

After dividing by $1-t^{2}$ we get

or, equivalently,

$$
\frac{s^{2}}{t^{2}}\left(1+t^{2}\right)+\frac{1+t^{2}+2 t^{4}}{t^{2}}=\frac{1-t^{2}}{s^{2}}\left(1+t^{2}\right)
$$

$$
\frac{s^{2}}{t^{2}}+\frac{1}{t^{2}}+\frac{2 t^{2}}{t^{2}+1}+\frac{t^{2}}{s^{2}}=\frac{1}{s^{2}} .
$$

But this is impossible since the LHS is greater than $\frac{s^{2}}{t^{2}}+\frac{t^{2}}{s^{2}} \geq 2$ and the RHS is $\leq 1$.

Thus we have two possibilities: either $s=0$ or $t^{2}=1$. Assume first that $t^{2}=1$. Then $t=-1$, and $k=2 s, l=1, m=2 s^{2}, n=s$. It is not difficult to check that in this case the $\tilde{S}$ matrix is symmetric. We have $-y_{1}=y_{1} x_{3}=\tilde{S}_{23}=$ $\frac{1}{\theta_{X} \theta_{Y}}\left(2 s \theta_{X} x_{1}+\theta_{Y} y_{1}\right)$. Recall that $x_{1}=-\frac{t}{t^{2}+1} y_{1}^{2}=\frac{1}{2} y_{1}^{2}$. Hence $s y_{1}=-\theta_{Y}-\frac{\theta_{Y}}{\theta_{X}}$. After applying the Galois automorphism we can assume that $y_{1}>0$ and we have an inequality $s y_{1} \leq 2$. On the other hand $y_{1}$ is a root of the polynomial $y^{2}-2 s y-2$ and hence $y_{1}^{2}=2 s y_{1}+2>2 s y_{1} \Rightarrow y_{1}>2 s$. Thus we get $2 s^{2}<1$ and hence $s=0$.

Consider now the case $s=0$. Then we have $k=0, l=1, m=0$. The $\tilde{S}$-matrix looks like

$$
\tilde{S}=\left(\begin{array}{ccc}
1 & 1 & y_{1} \\
1 & 1 & -y_{1} \\
y_{1} & y_{2} & 0
\end{array}\right)
$$

where $y_{1}, y_{2}$ are the roots of the equation $y^{2}=2+n y$. Since $\tilde{S}$ is symmetric we have $y_{2}=-y_{1}$ and hence $n=0$. Thus $K(\mathcal{C})=K(0,1,0,0)$.

Summarizing we can state

Proposition. Assume that a fusion category $\mathcal{C}$ of rank 3 admits a structure of modular category. Then we have the following possibilities for $K(\mathcal{C})$ : $K(\operatorname{Rep}(\mathbb{Z} / 3 \mathbb{Z})), K(1,0,0,0), K(0,1,0,1), K(1,1,1,0)$.

3.5. List of possible based rings. We have proved

Theorem. Assume that a fusion category of rank 3 admits a ribbon structure. Then we have the following possibilities for $K(\mathcal{C}): K(\operatorname{Rep}(\mathbb{Z} / 3 \mathbb{Z})), K(0,1,0,0)$, $K(0,1,0,1), K(1,1,1,0)$.

\section{IDENTIFICATION OF TENSOR CATEGORIES}

In this section we describe all fusion categories with Grothendieck rings given by Theorem 3.5.

4.1. $\quad K(\mathcal{C})=K(\operatorname{Rep}(\mathbb{Z} / 3 \mathbb{Z})$. In this case possible fusion categories are classified by $H^{3}\left(\mathbb{Z} / 3 \mathbb{Z}, k^{*}\right)=\mathbb{Z} / 3 \mathbb{Z}$ (note that $\operatorname{Aut}(\mathbb{Z} / 3 \mathbb{Z}$ ) acts trivially on this cohomology group, see e.g. [6]). Thus there are 3 such categories. But only the category with trivial associativity constraint admits a structure of ribbon category, see e.g. [16]. This structure is not unique: we can have symmetric category $\mathcal{C}=\operatorname{Rep}(\mathbb{Z} / 3 \mathbb{Z})$ and modular category $\operatorname{Rep}\left(\widehat{s l}(3)_{1}\right)$. 
4.2. $K(\mathcal{C})=K(0,1,0,0)$. The fusion ring $K(0,1,0,0)$ is well known in conformal field theory, it represents the fusion rules of the Ising model. We have an isomorphism $K(0,1,0,0)=K\left(\operatorname{Rep}\left(\widehat{s l}(2)_{2}\right)\right.$. Thus acoording to [9] (see also [1]) there are two fusion categories $\mathcal{C}$ such that $K(\mathcal{C})=K(0,1,0,0)$. One of them is $\operatorname{Rep}\left(\widehat{s l}(2)_{2}\right)$ and the second can be obtained from the first one by applying some Galois automorphism; also both categories can be constructed using the quantum group $U_{q}(\operatorname{sl}(2))$ for $q=\sqrt[8]{1}$, see [1].

4.3. $K(\mathcal{C})=K(1,1,1,0)$. Observe that $K(1,1,1,0) \otimes K(\operatorname{Rep}(\mathbb{Z} / 2 \mathbb{Z}))=K\left(\operatorname{Rep}\left(\widehat{s l}(2)_{5}\right)\right.$. Thus it follows from [9] (see also [1]) that there are exactly three fusion categories $\mathcal{C}$ with such Grothendieck ring; one category is a subcategory $\operatorname{Rep}\left(\widehat{s o}(3)_{5}\right)$ of representations with integer spin in $\operatorname{Rep}\left(\widehat{s l}(2)_{5}\right)$; two others are Galois conjugate to this one. Thus all three categories admit a ribbon structure. Also all three categories can be realized using the quantum group $U_{q}(s l(2))$ for $q=\sqrt[7]{1}$, see [1].

4.4. $K(\mathcal{C})=K(0,1,0,1)$. Observe that $K(0,1,0,1)=K\left(\operatorname{Rep}\left(S_{3}\right)\right)$. It was established by T. Chmutova that there are 3 fusion categories with such Grothendieck ring, see [6]. It is easy to see that $\tilde{S}$-matrix for such category necessarily has rank 1 and hence any braided structure on $\mathcal{C}$ is symmetric. Thus only $\mathcal{C}=\operatorname{Rep}\left(S_{3}\right)$ of these 3 categories has a ribbon structure.

4.5. Summarizing the results of the previous sections we can state the main result of this note.

Main Theorem. There are exactly 7 fusion categories of rank 3 admitting a ribbon structure: $\operatorname{Rep}(\mathbb{Z} / 3 \mathbb{Z}), \operatorname{Rep}\left(S_{3}\right), \operatorname{Rep}\left(\widehat{s l}(2)_{2}\right), \operatorname{Rep}\left(\widehat{s o}(3)_{5}\right)$ and the Galois conjugates of two latter categories.

Remark. It is interesting to note that there exists a fusion category of rank 3 (and hence with commutative Grothendieck ring) which does not admit a ribbon structure. Namely let $\mathcal{C}$ be the fusion category attached to the affine $s l_{2}$ on level 10 and let $A \in \mathcal{C}$ be the commutative $\mathcal{C}$-algebra of type $E_{6}$, see [12. Then the category $\operatorname{Rep} A$ of right $A$-modules has a structure of fusion category, see loc. cit. This fusion category contains a tensor subcategory corresponding to the ends of long legs and the triple vertex of the graph $E_{6}$ which is fusion category of rank 3 with Grothendieck ring $K(0,1,0,2)$. It follows from the Main Theorem above that this category does not admit a ribbon structure.

It seems reasonable to expect that the Grothendieck ring of any fusion category of rank 3 is either isomorphic to $K(0,1,0,2)$ or listed in Theorem 3.5 .

\section{REFERENCES}

[1] B. Bakalov, A. Kirillov, Jr., Lectures on tensor categories and modular functor, Amer. Math. Soc., 2000

[2] M. Caselle, G. Ponzano Modular invariance and fusion rule algebras: classification of the three-operator case, Internat. J. Modern Phys. A 5 (1990), no. 24, 4697-4719.

[3] A. Coste, T. Gannon, Remarks on Galois symmetry in rational conformal field theories, Phys. Lett. B 323 (1994), no. 3-4, 316-321.

[4] J. de Boere, J. Goeree, Markov traces and II 1 factors in conformal field theory, Comm. Math. Phys. 139 (1991), no. 2, 267-304.

[5] P. Deligne, Catégories tensorielles, Moscow Math. Journal 2 (2002) no. 2, 227-248.

[6] P. Etingof, S. Gelaki, V. Ostrik, Classification of fusion categories of dimension pq, Int. Math. Res. Not. 2004, no. 57, 3041-3056.

[7] P. Etingof, D. Nikshych, V. Ostrik, On fusion categories, preprint math.QA/0203060 
[8] P. Etingof, On Vafa's theorem, Math. Res. Lett. 9 (2002), no. 5-6, 651-657.

[9] J. Frölich, T. Kerler, Quantum groups, quantum categories and quantum field theory, LNM 1542 .

[10] D. Gepner, A. Kapustin, On the classification of fusion rings, Phys. Lett. B 349 (1995), no. $1-2,71-75$.

[11] D. Kazhdan, H. Wenzl, Reconstructing monoidal categories, I. M. Gelfand Seminar, 111-136, Adv. Soviet Math., 16, Part 2, Amer. Math. Soc.

[12] A. Kirillov, V. Ostrik, On q-analog of McKay correspondence and ADE classification of conformal field theories, Adv. Math. 171 (2002), no. 2, 183-227.

[13] E. Landau, Über die Klassenzahl der binären quadratischen Formen von negativer Discriminante, Math. Ann. 56 (1903), 671-676.

[14] M. Müger, On the structure of modular categories, Proc. London Math. Soc. (3) 87 (2003), no. 2, 291-308.

[15] V. Ostrik, Fusion categories of rank 2, Math. Res. Lett. 10 (2003), no. 2-3, 177-183.

[16] F. Quinn, Group categories and their field theories, Geometry \& Topology Monographs, Volume 2: Proceedings of the Kirbyfest, 407-453.

[17] C. Vafa, Toward classification of conformal theories, Phys. Lett. B 206 (1988),421-426.

E-mail address: vostrik@darkwing.uoregon.edu

Department of Mathematics, 1222 University of Oregon, Eugene OR 97403-1222 